\title{
Diagnostic Value of SCD163 Combined with PCT and HS-CRP in Patients with Gynecological Malignant Tumors and Fever
}

\author{
Weiwei Shen ${ }^{1}$ and Shiping Lai ${ }^{2}$ \\ ${ }^{1}$ Department of Obstetrics and Gynecology, Huzhou Maternity \& Child Health Care Hospital, Zhejiang Province, China \\ ${ }^{2}$ Department of Pathology, Huzhou Maternity \& Child Health Care Hospital, Zhejiang Province, China
}

\begin{abstract}
Objective: To explore diagnostic values of SCD163 with PCT and HS-CRP in diagnosing infection among patients with gynecological malignant tumors and fever.

Study Design: Cross-sectional, observational study.

Place and Duration of Study: Huzhou Maternity \& Child Health Care Hospital, China, from May 2018 to February 2020.

Methodology: A total of 119 patients with gynecological malignant tumors and fever were divided into infected group (70 cases) and non-infected group (49 cases), according to the blood bacterial culture results and clinical symptoms. Serum PCT, HS-CRP, SCD163 level were compared. Pearson correlation was performed to analyse correlation of SCD163 with PCT and HSCRP. The area under the curve (AUC) score was calculated from receiver operating characteristic curve (ROC).

Results: Serum SCD163, PCT and HS-CRP levels in infected group were all elevated compared to non-infected group (all $p$ $<0.001)$. Pearson correlation analysis showed that SCD163 was positively correlated with PCT and HS-CRP $(r=0.596, p<0.001$; $r=0.744, p<0.001$, respectively). The AUC of sCD163 + PCT + HS-CRP combined detection for predicting early infection in patients with gynecological malignant tumors and fever was 0.905 (95\% Cl: 0.855-0.956), which was greater than sCD163, PCT, and HS-CRP detected alone.

Conclusion: Combined detection of SCD163 with PCT and HS-CRP has a high diagnostic efficiency for early infection in patients with gynecological malignant tumors and fever, which is better than that of SCD163, PCT or HS-CRP alone. It can be used as an effective auxiliary examination method and an indicator for differential diagnosis.
\end{abstract}

Key Words: SCD163, PCT, HS-CRP; Gynecology, Tumor, Infection, Diagnosis.

How to cite this article: Shen W, Lai S. Diagnostic Value of SCD163 Combined with PCT and HS-CRP in Patients with Gynecological Malignant Tumors and Fever. J Coll Physicians Surg Pak 2020; 30(10):1053-1057.

\section{INTRODUCTION}

Fever is one of the common clinical symptoms; and sometimes the first and the main symptom of malignant tumors. ${ }^{1}$ Fever in tumor patients can be due to infectious and non-infectious causes; non-infectious fever accounts for over $23 \%$ of fever cases in tumor patients. ${ }^{2}$ For patients with malignant tumors, in addition to fever-producing infections like bacteria and fungi, the tumor cells themselves can also release pyrogenic factors and cause non-infectious fever. ${ }^{3}$ In addition, use of chemotherapy drugs and blood products or other non-infectious factors can also cause fever. ${ }^{4,5}$

Correspondence to: Dr. Shiping Lai, Department of Pathology, Huzhou Maternity \& Child Health Care Hospital, Zhejiang Province, China

E-mail: uupnm1@163.com

Received: May 20, 2020; Revised: August 17, 2020;

Accepted: August 17, 2020

DOI: https://doi.org/10.29271/jcpsp.2020.10.1053
The difficulty in distinguishing between these causes often leads to the irrational use of anti-infective agents that will exacerbate the propagation of resistant bacteria and increase the possibility of multiple infections. Therefore, it is of great importance to quickly and accurately diagnose early infections in patients with malignant tumors and fever.

Blood culture is the gold standard for definitive diagnosis of infections. However, it takes long with a low positive rate. ${ }^{6}$ Routine hematology indicators are often affected by factors such as corticosteroids or chemotherapy use, and bone marrow status of tumor patients, showing poor sensitivity and specificity. As a precursor protein of calcitonin, procalcitonin (PCT) is commonly used as a serum marker in the diagnosis and differential diagnosis of systemic bacterial infections in patients. However, PCT levels in patients with gram-negative bacterial infections are significantly higher than those in gram-positive bacterial infections, thus limiting its role in monitoring gram-positive bacterial infections. ${ }^{7}$ High-sensitivity C-reactive protein (HS-CRP) is widely used in the early detection and diagnosis of various diseases including those related to infection. 
But as it is easily affected by multi-system disease factors, such as those of the digestive system, cardio-cerebrovascular and endocrine system, etc., its sensitivity and specificity in the diagnosis of deep fungal infections are low. ${ }^{8}$ Therefore, it is particularly important to select a convenient and effective indicator for detecting infection.

Hemoglobin scavenger receptor (CD163) is a transmembrane molecule found exclusively on the cell membrane of the monocyte-macrophages system so far. Endotoxin can induce CD163 to shed and become soluble CD163 (sCD163). ${ }^{9}$ Under the effect oftumors, infections and otherdiseases, macrophages are overexpressed, CD163 is shed into the blood or tissue fluid, and the level of sCD163 will be significantly increased. This is a marker of macrophage activation. ${ }^{10} \mathrm{~A}$ study has shown that sCD163 may be a sensitive indicator of early sepsis, and the changes in its levels can be used as early predictors of organ failure. ${ }^{11}$ One study has also demonstrated that serum sCD163 levels in patients with severe bacterial infections are elevated compared to normal controls. ${ }^{12}$ At present, there are few reports on the diagnostic value of SCD163 combined with PCT and HSCRP in infection occurring in patients with gynecological malignant tumors and fever.

The purpose of this study was to explore the diagnostic value of combined detection of SCD163 with PCT and HS-CRP in infection among patients with gynecological malignant tumors and fever.

\section{METHODOLOGY}

This research was carried out in the Huzhou Maternity \& Child Health Care Hospital, China, from May 2018 to February 2020. This study was approved by the hospital Ethics Committee. A total of 119 patients with gynecological malignant tumors and fever were selected as the research subjects. The diagnostic criteria for fever were axillary body temperature $>38^{\circ} \mathrm{C}$ for over 6 hours or $>38.5^{\circ} \mathrm{C}$ for one time. The diagnostic criteria for infection were the corresponding clinical manifestations and signs; and/or imaging examination clearly identifying the focus of infection; and/or a diagnostic basis in etiology or serum immunology. Inclusion criteria were those diagnosed as malignant tumors based on pathological or cytological diagnostic criteria with fever; not being already treated with antimicrobial agents; and patients aged $\geq 18$ years old. Exclusion criteria were patients aged $<18$ years old; those who died within 24 hours of enrollment; patients with agranulocytosis $\left(<0.5 \times 10^{9} / \mathrm{L}\right)$; patients with HIV infection, type 2 diabetes, viral hepatitis, and autoimmune diseases; patients with blood bacterial culture results suspected of contamination; non-gynecological malignant tumor patients; mental illness patients; and fever patients induced by non-infectious factors such as allergic reactions, chemotherapy drugs or blood products.

Blood bacterial culture was performed for all patients before treatment, and they were divided into the infected group (70 cases) and the non-infected group (49 cases), according to the blood bacterial culture results and clinical symptoms.
The $5 \mathrm{~mL}$ fasting cubital venous blood was collected from all objects, centrifuged, and the upper serum was taken. A nephelometric analyser (Siemens, $\mathrm{BN}^{\mathrm{TM}}$ II System) was used to determine the CRP level; and a tubular chemiluminescence analyser (Nanjing Norman Biological Technology, Norman-100) was used to determine the PCT level. Serum PCT level was determined by chemiluminescence immune assay; HS-CRP level by high-sensitivity immunoturbidimetry; and serum SCD163 level by doubleantibody sandwich ELISA.

SPSS version 25.0 statistical software was used for analysis. Enumeration data was expressed in $n(\%)$. Kolmogorov-Smirnov test and Shapiro-Wilk test were used for the evaluation of the normality of measurement data. Measurement data with normal distribution were expressed by Mean \pm SD and independent sample t-test was conducted. Pearson correlation was performed to analyse the correlation of SCD163 with PCT and HS-CRP.

The area under the curve (AUC) score was calculated from the receiver operating characteristic curve (ROC). The $p<0.05$ was statistically significant.

\section{RESULTS}

Among the 119 patients, all were females, aged 34-71 (62.38 \pm 3.56) years; the body temperature was 38.5-41.5 (39.54 \pm $0.72)^{\circ} \mathrm{C}$; there were 37 cases of ovarian cancer $(31.09 \%), 43$ cases of cervical cancer (36.13\%), and 39 cases of endometrial cancer $(32.77 \%)$.

Serum SCD163, PCT and HS-CRP levels in the infected group were all elevated compared to the non-infected group (all $p$ $<0.001$, Table I). Pearson correlation analysis of serum SCD163 with PCT and HS-CRP was performed in the infected group. The results showed that $\mathrm{SCD} 163$ was positively correlated with PCT and HS-CRP ( $r=0.596, p<0.001 ; r=0.744, p<0.001$, respectively, as shown in Figure 1 ).

Table I: Comparison of serum sCD163, PCT and HS-CRP levels between the twogroups.

\begin{tabular}{|l|c|c|c|}
\hline Parameter & $\begin{array}{c}\text { Infected group } \\
(\mathbf{n = 7 0 )}\end{array}$ & $\begin{array}{c}\text { Non-infected group } \\
(\mathbf{n = 4 9 )}\end{array}$ & p-value \\
\hline sCD163 $(\mathrm{pg} / \mathrm{mL})$ & $137.24 \pm 9.86$ & $94.54 \pm 4.64$ & $<0.001$ \\
\hline PCT $(\mathrm{ng} / \mathrm{mL})$ & $11.66 \pm 1.21$ & $0.71 \pm 0.10$ & $<0.001$ \\
\hline HS-CRP $(\mathrm{mg} / \mathrm{L})$ & $88.78 \pm 4.33$ & $58.86 \pm 2.43$ & $<0.001$ \\
\hline
\end{tabular}

The AUC of sCD163 was $0.824(95 \% \mathrm{Cl}: 0.752-0.897)$, the optimal cut-off value $98.78 \mathrm{pg} / \mathrm{mL}$, sensitivity $85.90 \%$, and specificity $61.80 \%$. The AUC of PCT was $0.740(95 \% \mathrm{Cl}$ : $0.650-0.830$ ), the optimal cut-off value $10.40 \mathrm{ng} / \mathrm{mL}$, sensitivity $70.30 \%$, and specificity $74.50 \%$. The AUC of HS-CRP was 0.818 (95\% Cl: $0.743-0.893)$, the optimal cutoff value $61.42 \mathrm{mg} / \mathrm{L}$, sensitivity $90.60 \%$, and specificity $56.40 \%$.

The AUC of SCD163 + PCT + HS-CRP combined detection for predicting early infection in patients with gynecological malignant tumors and fever was 0.905 (95\% Cl: 0.855-0.956), sensitivity $90.60 \%$, and specificity $70.90 \%$. The AUC of combined detection was greater than SCD163, PCT, and HS-CRP detected alone, as shown in Table ll and Figure 2. 
Table II: ROC curve analysis results.

\begin{tabular}{|l|c|c|c|c|}
\hline Parameter & SCD163 & PCT & HS-CRP & SCD163+PCT+HS-CRP \\
\hline AUC & 0.824 & 0.740 & 0.818 & 0.905 \\
\hline $95 \% \mathrm{Cl}$ & $0.752-0.897$ & $0.650-0.830$ & $0.743-0.893$ & $0.855-0.956$ \\
\hline Sensitivity (\%) & 85.90 & 70.30 & 90.60 & 90.60 \\
\hline Specificity (\%) & 61.80 & 74.50 & 56.40 & 70.90 \\
\hline
\end{tabular}

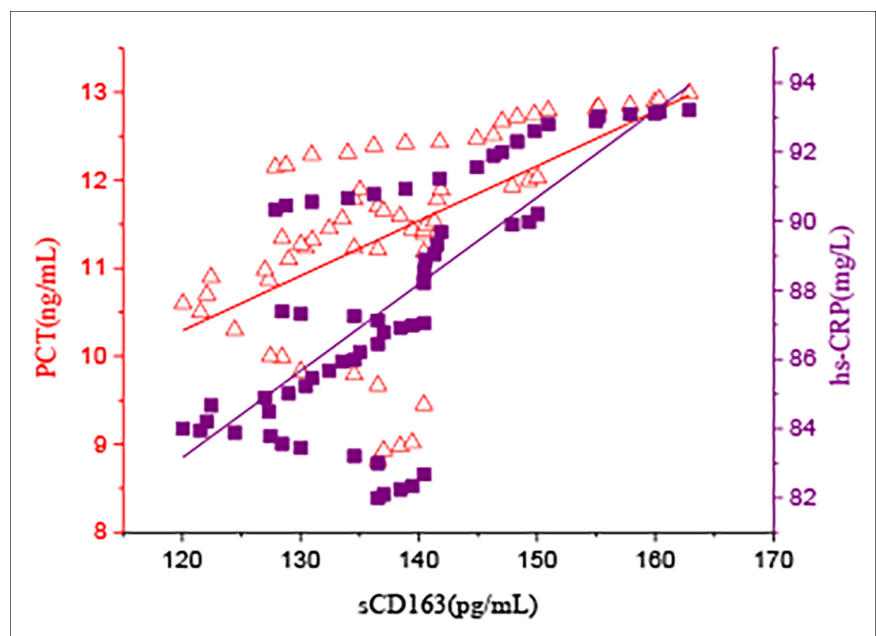

Figure 1: Correlation between serum SCD163 and PCT (red line, $r=$ $0.596, p<0.001$ ), HS-CRP (purple line, $r=0.744, p<0.001$ ) in the infected group $(n=70)$.

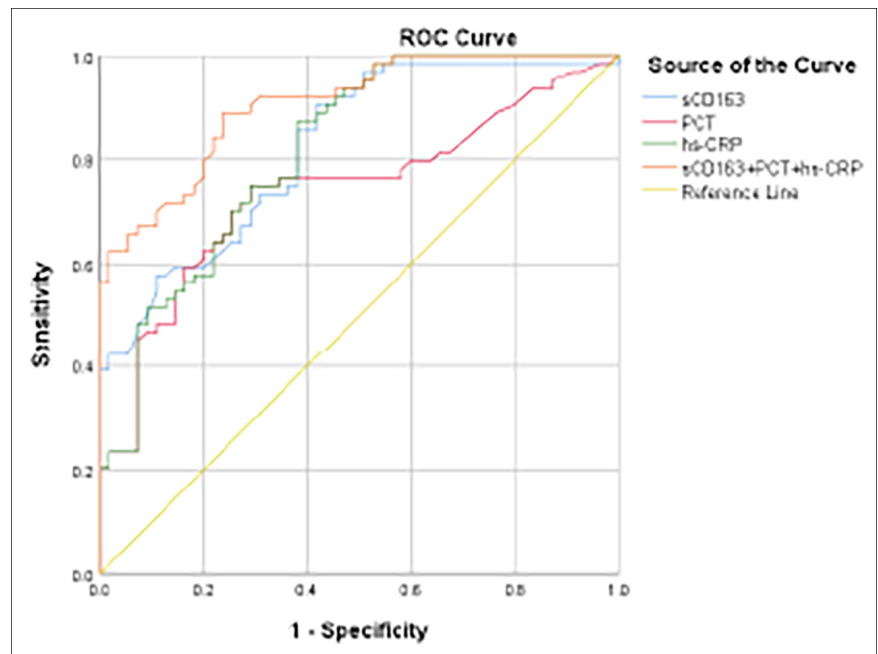

Figure 2: ROC curves of early detection of SCD163, PCT, HS-CRP and the combined detection of patients with gynecological malignant tumors and fever.

\section{DISCUSSIONS}

Studies have demonstrated that PCT level is low in normal organisms, but it will experience a rapid rise in the presence of infections caused by fungi or bacteria, and it is positively correlated with the degree of infection. ${ }^{13}$ When patients' infection is controlled, PCT level is significantly reduced with relatively higher specificity and sensitivity. Under the action of fungi, bacteria and other pathogenic bacteria, PCT level increases significantly. It is of important clinical value in the diagnosis of bacterial and non-bacterial infections. ${ }^{14}$
CRP is an acute phase reaction protein. It is synthesised by the liver in the early stress response to severe factors. Its level will rise rapidly at the later stage of infections, and is positively correlated with the degree of infections. It is a marker of systemic inflammatory response. ${ }^{15}$ An HS-CRP test is more sensitive than a standard test for detecting CRP. ${ }^{16}$

SCD 163 is a monocyte- and macrophage-specific scavenger receptor, and an important marker of macrophage activation. ${ }^{17}$ Studies have reported that the inflammatory response can stimulate the massive release of SCD163, whose role is important in inflammatory and infectious diseases. ${ }^{18}$

This study revealed that the levels of SCD163, PCT and HSCRP in the infected group are significantly elevated compared to the non-infected group. This indicates that sCD163, PCT and HS-CRP levels are elevated when patients with gynecological malignant tumors and fever become infected. SCD163, PCT and HS-CRP play a major role in monitoring infectious diseases. Su et al. has demonstrated that serum soluble trigger receptors SCD163 and PCT can be used to predict the prognosis of patients with sepsis. ${ }^{19}$

Knudsen et al. has reported that PCT with a high diagnostic value as a marker of bacterial infection is superior to sCD163; however, sCD163 may help identify patients quickly with systemic bacterial infection. ${ }^{20}$ Research by Zakariah et al. has shown that HS-CRP is a sensitive marker for diagnosing infected diabetic foot ulcer. ${ }^{21}$

ROC analysis is used to evaluate the test efficacy of SCD163 combined with commonly used clinical infection markers PCT and HS-CRP. The results show that the AUC of SCD163 combined with PCT and HS-CRP is significantly larger than that of SCD163, PCT or HS-CRP alone, and the sensitivity and specificity are higher. It suggests that the combined detection of SCD163 with PCT and HS-CRP has a high diagnostic value for early infection.

There are some limitations to be noted. The gynecological malignant tumors included in this study are limited to ovarian cancer, cervical cancer, and endometrial cancer. This study is a retrospective analysis with small sample size, and further large-sample prospective studies are still needed to confirm the results. In the infected group, the coexistence of infectious fever and non-infectious fever cannot be ruled out completely. As blood bacterial culture is affected by many factors, there may be false negative 
patients with blood-borne infections included in the non-infected group.

\section{CONCLUSION}

The combined detection of SCD163 with PCT and HS-CRP has a high diagnostic efficiency for early infection in patients with gynecological malignant tumors and fever, which is better than that of SCD163, PCT or HS-CRP alone. It can be used as an effective auxiliary examination method and an indicator for differential diagnosis.

\section{ETHICAL APPROVAL:}

This study has been approved by the Ethics Committee of Huzhou Maternity and Child Health Care Hospital, China.

\section{PATIENTS' CONSENT:}

Informed consents were obtained from all participants.

\section{CONFLICT OF INTEREST:}

Authors declared no conflict of interest.

\section{AUTHORS' CONTRIBUTION:}

WS: Contributed to design articles, collected and analysed data, and wrote the manuscript.

SL: Revised the manuscript and approved the final manuscript.

\section{REFERENCES}

1. Pasikhova Y, Ludlow S, Baluch A. Fever in patients with cancer. Cancer Control 2017; 24(2):193-7. doi: 10.1177/107327 481702400212.

2. Toussaint E, Bahel-Ball E, Vekemans M, Georgala A, AlHakak L, Paesmans $M$, et al. Causes of fever in cancer patients (prospective study over 477 episodes). Support Care Cancer 2006; 14(7):763-9. doi: 10.1007/s00520005-0898-0.

3. Eggimann P, Glauser MP, Aoun M, Meunier F, Calandra T. Cefepime monotherapy for the empirical treatment of fever in granulocytopenic cancer patients. J Antimicrob Chemother 1993; 32 Suppl B:151-63. doi: 10.1093/jac/ 32.suppl_b.151.

4. Grützmeier S, von Schenck H. C-reactive protein during chemotherapy for acute leukemia with special reference to non-infective causes of fever. Med Oncol Tumor Pharmacother 1986; 3(7):71-5. doi: 10.1007/BF02934556.

5. Maheshwari V, Kaore NM, Ramnani VK, Sarda S. A comparative evaluation of different diagnostic modalities in the diagnosis of typhoid fever using a composite reference standard: A tertiary hospital based study in central india. J Clin Diagn Res 2016; 10(10):DC01-DC04. doi: 10.7860/ JCDR/2016/20426.8684.

6. Zhou L】Pollard1 AJ. A fast and highly sensitive blood culture PCR method for clinical detection of Salmonella enterica serovar Typhi. Ann Clin Microbiol Antimicrob 2010; 9: 14. doi: 10.1186/1476-0711-9-14.

7. Murri R, Mastrorosa I, Taccari F, Baroni S, Giovannenze F,
Palazzolo $C$, et al. Procalcitonin is useful in driving the choice of early antibiotic treatment in patients with bloodstream infections. Eur Rev Med Pharmacol Sci 2018; 22(10):3130-7. doi: 10.26355/eurrev_201805_15072.

8. Yao A, Liu J, Chang J, Deng C, Hu Y, Yu F, et al. Clinical practice of procalcitonin and hypersensitive c-reactive protein test in neonatal infection. Pak J Pharm Sci 2016; 29(2 Suppl):753-6.

9. Thornton S, Tan R, Sproles A, Do T, Schick J, Grom AA, et al. A Multiparameter flow cytometry analysis panel to assess cd163 mrna and protein in monocyte and macrophage populations in hyperinflammatory diseases. J Immunol 2019; 202(5):1635- 43. doi: 10.4049/jimmunol. 1800765.

10. Rittig N, Svart M, Jessen N, Møller N, Møller HJ, Grønbæk H. Macrophage activation marker SCD163 correlates with accelerated lipolysis following LPS exposure: A humanrandomised clinical trial. Endocr Connect 2018; 7(1):107-114. doi: 10.1530/EC-17-0296.

11. Piatkowski A, Grieb G, Das R, Bozkurt A, Ulrich D, Pallua N. Soluble CD163: A novel biomarker for the susceptibility to sepsis in severe burn injuries. Indian J Plast Surg 2011; 44(1):118-24. doi: 10.4103/0970-0358.81454.

12. Schaer DJ, Schleiffenbaum B, Kurrer M, Imhof A, Bächli E, Fehr J, et al. Soluble hemoglobin-haptoglobin scavenger receptor CD163 as a lineage-specific marker in the reactive hemophagocytic syndrome. Eur J Haematol 2005; 74(1):6-10. doi: 10.1111/j.1600-0609.2004.00318.x.

13. Hu C, Jiang J1, Li Z1, Li Y. Expression pattern of soluble triggering receptor expressed on myeloid cells-1 in mice with acinetobacter baumannii colonization and infection in the lung. J Thorac Dis 2018; 10(3):1614-21. doi: 10.21037/ jtd.2018.03.41.

14. Memar MY, Varshochi M, Shokouhi B, Asgharzadeh M, Kafil HS. Procalcitonin: The marker of pediatric bacterial infection. Biomed Pharmacother 2017; 96:936-43. doi: 10.1016/j.biopha.2017.11.149.

15. Igitova MB, Serdiuk GV, Momot AP, Vorob'eva EN. The clinical and diagnostic value of C-reactive protein as a marker of a systematic inflammatory response in pregnancy (a review of literature). Klin Lab Diagn 2009; 6:3-8.

16. Hudzik B, Szkodzinski J, Pietka-Rzycka A, Danikiewicz A, Wojnar R, Lekston A, et al. Plasma pentraxin 3 may be a more sensitive marker of inflammatory response than high-sensitivity C-reactive protein after bare-metal stent compared to drug-eluting stent implantation. J Interferon Cytokine Res 2013; 33(5):280-4. doi: 10.1089/jir.2012. 0023.

17. Azuma Y, Suzuki Y, Okada S, Matsuguma C, Wakiguchi H, Ohnishi Y, F et al. Utility of soluble CD163 in the clinical management of patients with kawasaki disease. Front Pediatr 2020; 8:148. doi: 10.3389/fped.2020.00148.

18. Suzuki $Y$, Enomoto $Y$, Yokomura K, Hozumi H, Kono M, Karayama $M$, et al. Soluble hemoglobin scavenger receptor CD163 (sCD163) predicts mortality of community-acquired pneumonia. J Infect 2016; 73(4):375-7. doi: 
10.1016/ j.jinf.2016.07.004.

19. Su L, Feng L, Song Q, Kang H, Zhang X, Liang Z, et al. Diagnostic value of dynamics serum SCD163, sTREM-1, PCT, and CRP in differentiating sepsis, severity assessment, and prognostic prediction. Mediators Inflamm 2013; 969875. doi: 10.1155/2013/969875.

20. Knudsen TB, Larsen K, Kristiansen TB, Møller HJ, Tvede M, Eugen-Olsen J, et al. Diagnostic value of soluble CD163 serum levels in patients suspected of meningitis: comparison with CRP and procalcitonin. Scand J Infect Dis 2007; 39(6-7):542-53. doi: 10.1080/0036554060 1113685.

21. Zakariah NA, Bajuri MY, Hassan R, Ismail Z, Mansor M, Othman $\mathrm{H}$, et al. Is Procalcitonin more superior to HS-CRP in the diagnosis of infection in diabetic foot ulcer? Malays J Pathol 2020; 42(1):77-84. 